\title{
Calcification detection for intravascular ultrasound image using direct acyclic graph architecture: pre-trained model for 1-channel image
}

\author{
Hannah Sofian', Joel Chia Ming Than², Suraya Mohamad ${ }^{3}$, Norliza Mohd Noor ${ }^{4}$ \\ ${ }^{1}$ Universiti Kuala Lumpur Malaysian Institute Information Technology (UniKL MIIT), Malaysia \\ ${ }^{1,4}$ Razak Faculty of Technology and Informatics, Universiti Teknologi Malaysia (UTM), Malaysia \\ ${ }^{2}$ School of Information and Communication Technologies, Faculty of Engineering, Computing and Science, Swinburne \\ University of Technology Sarawak Campus, Malaysia \\ ${ }^{3}$ Universiti Kuala Lumpur British Malaysian Institute (UniKL BMI), Malaysia
}

\begin{tabular}{l} 
Article Info \\
\hline Article history: \\
Received Jan 5, 2021 \\
Revised Feb 9, 2021 \\
Accepted Feb 21, 2021 \\
\hline Keywords: \\
Calcification \\
Coronary artery disease \\
Direct acyclic graph \\
Transfer learning \\
Transformed Image
\end{tabular}

Article Info

\begin{abstract}
Coronary artery calcification is a calcium buildup within the walls of the arteries. It is considered a predominant marker for coronary artery disease. Thus many approaches have been developed for the automatic detection of calcification. The previous calcification detection was on segmentation of other structures as pre-processing steps or using the fact that the calcification often appears as a bright region. In this paper, an automated system proposed using a deep learning approach to detect the calcification absence and calcification presence in coronary artery IVUS image. A useful advantage of deep learning, compared to other methods is, it uses representations and features directly from the raw data, bypassing the need to manually extract features, a common that required in the traditional machine learning framework. The type of deep learning architecture used is 27 layers of convolutional neural networks (CNNs) using direct acyclic graph. The proposed system used 2175 images and achieved an accuracy of $98.16 \%$ for Cartesian coordinate images and $99.08 \%$ for polar reconstructed coordinate images.
\end{abstract}

This is an open access article under the CC BY-SA license.

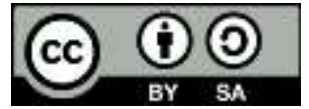

\section{Corresponding Author: \\ Hannah Sofian \\ Universiti Kuala Lumpur Malaysian Institute Information Technology (UniKL MIIT) \\ Kuala Lumpur Malaysia \\ Email: hannah@unikl.edu.my}

\section{INTRODUCTION}

Cardiovascular disease (CVD) involved disease that affected the heart and the blood vessel, such as coronary artery disease and stroke. Other cardiovascular diseases type related to the heart are hypertensive, inflammatory and rheumatic disease [1]. Cardiovascular diseases (CVD) are the leading non-communicable diseases (NCDs) that cause death [2]. The estimation of death in 2016 was 41 million, equivalent to $71 \%$ for all deaths globally caused by NCDs. Out of these, 17.9 million deaths (44\%) are due to CVD, 9.0 million deaths $(22 \%)$ are due to cancer, 3.8 million deaths $(9 \%)$ are due to chronic respiratory disease, and 1.6 million deaths $(4 \%)$ are due to diabetes. Coronary artery calcification is a calcium buildup within the wall of the arteries that supply oxygen-rich blood to the heart. This calcium causes the walls to become more hardened, as seen with atherosclerosis. Coronary artery calcification is highly prevalent in patients with coronary heart disease and is associated with adverse cardiovascular events [3], [4]. Besides, the presence or absence of calcium, shown to be the determinant of the percutaneous coronary intervention (PCI) success [5]. 
In Figure 1(a), the author illustrates the coronary artery cross-section to show the four main structures, namely the tunica adventitia, tunica media, tunica intima and lumen. Figure 1(b) shows the original image of the intravascular ultrasound (IVUS) image in portable network graphic (PNG) format, while Figure 1(c) shows the transformed image (polar reconstructed image) of Figure 1(b). In the IVUS image, Coronary artery calcification appears as a bright hyperechoic (blue arrow) compared to the adventitia and often appear with a corresponding dark shadow artefact (yellow arrow) behind the tunica media and tunica adventitia.

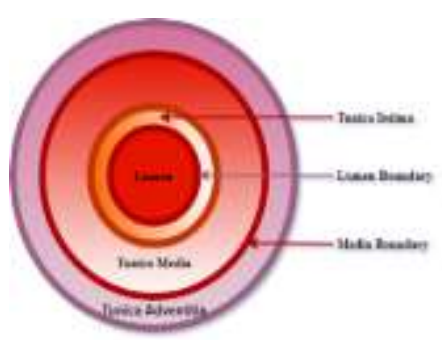

(a)

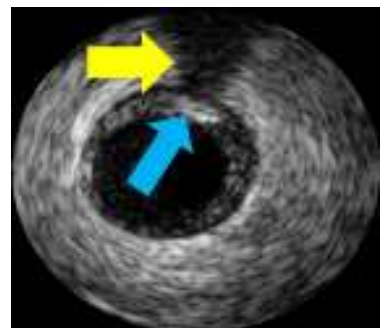

(b)

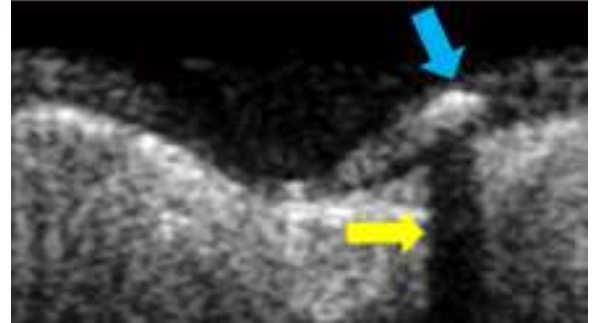

(c)

Figure 1. (a) The illustration of the cross-section layers of the blood vessel wall by the author (b) and sample of coronary artery IVUS image with calcification presense in cartesian coordinate image (c) and in polar reconstructed coordinate image

The study to detect calcification on intravascular ultrasound images have been done by many researchers. The methods used for calcification detection involve various segmentation and feature extraction based approaches in the cartesian coordinate image and polar reconstructed coordinate image of the coronary artery. Since calcification often found in the surrounding area near tunica lumen and tunica adventitia, many approaches often perform segmentation of these regions before performing the calcification detection. Work in [6] used 60 IVUS images from 7 patients as the test images to detect calcification. All the image converted into the polar reconstructed coordinate image. Then parametric deformable models and geometric deformable models were used to detect the lumen and media adventitia border. Then bayesian classifier is used to find calcification inside the region between the lumen and the adventitia boundary. The result obtained is good, with a score of above $90 \%$. Similar work is proposed by [7] using fuzzy k-means. Another study that worked is described in [8]. The proposed method first segmented was the coronary wall and plaque. Then, the proposed method continued with the determination for plaque composition to one of the main three classes: soft plaque, hardened plaque or, hard plaque shadow.

Another main important characteristic of the calcification is that the calcification regions often represent high-intensity echo in IVUS images. This fact makes it possible to segment the calcified region area by using gray-level threshold techniques. Adaptive threshold (otsu method), followed by morphological operation, then an empirical threshold value was proposed by [9] to segment the calcification boundary. The second work that utilized the otsu thresholding technique is [10]. Their method was further improving by introducing an additional procedure that can eliminate wrongly segmented bright regions, which is quite similar in appearance to calcification. Furthermore, the detection of an acoustic shadow performed well. Twenty images obtained were from different patients, 15 images presented with calcification and five images without calcification. This results obtained were $88 \%$ specificity and $84 \%$ sensitivity.

All work of the aforementioned required performing segmentation of the region of interest before calcification detection. In recent years, calcification detection was by assessing the overall appearance of an IVUS image. Using this approach of a unique image pattern extracted from the image and reasoning is made to identify the relationship between the perceived patterns and possible diagnosis. This work has the advantages of not relying on the result of the segmentation of other regions. As mentioned earlier, the calcification presence in IVUS can see when a dark shadow artefact appeared behind the calcification. The presence of the calcification and the dark shadow artefact will make the media boundary segmentation very challenging.

Coarse to fine was a strategy by [11] to detect the calcification region. The method first used rayleigh mixture model (RMM) for pixel classification. Then the detection of angular location of the calcification using markov random field (MRF) was performed. The next step was a refinement of the detected calcification region by five predefined constraints. Lastly, the graph searching algorithm was to identify the final calcification border. A total of 996 images from eight patients had used in this study. The 
images are composed of 498 with calcified plaque and another 498 without calcified plaque. The results obtained was a good result with a score of above $94 \%$.

The deep learning method is the current technology widely used using the concept of neural network and machine learning for medical imaging images. The main advantage of deep learning is the computers learn useful representations and features directly from the raw data, bypassing the need to manually extract features required in the traditional machine learning framework [12]. The application using deep learning methods had triggered the researchers to use deep learning to analyze the images [13], [14]. Deep learning is becoming popular among medical researchers in medical imaging, such as detecting cancer cells, eye disease, lung disease and coronary artery disease. The types of CNNs network used varies between different applications. For example, a pre-trained CNN network of VGG16 [15] and, InceptionResNet-V2, InceptionV3 and, ResNet-50 architecture [16] had used to detect diabetic macular edema (DME). AlexNet, VGG19 and, Inception- V3 architecture had used to detect kawasaki disease in optical coherence tomography (OCT) images [17]. AlexNet, VGGNet, GoogleNet and ResNet architecture had used to detect breast cancer using mammogram images [18]. AlexNet architecture alone also used to find the thorax disease in Lung using an X-Ray [19] and a tumour from the breast mammography images [20] and AlexNet, ResNet-101 and InceptionResNet-V2 architecture had used to detect calcification in Cartesian coordinate IVUS images and Polar reconstructed coordinate IVUS images [21].

Due to the effectiveness of using deep learning for medical image classification, in this paper we described a framework for calcification detection using deep learning, specifically proposed 27 layers of convolutional neural networks (CNNs) using direct acyclic graph and transfers learning. The 27 layers CNNs consists of an input max-pooling, batch normalization and rectified linear unit (ReLu), 6-convolutional layers with max-pooling, batch normalization and ReLu and 1-Fully connected layer to detect calcification in intravascular ultrasound (IVUS) image. The approach will classify the images into one of these two classes, the images with calcification and images without calcification. To our knowledge, the direct acyclic graph CNN has not applied to IVUS image for calcification detection in any previous works.

\section{MATERIALS AND METHOD}

\subsection{Material}

The image dataset used is obtained from dataset B from MICCAI challenge 2011. It consists of 2175 images from 10 patients, where 1645 are images with plaque composites, and another 530 images are with composites plaque and calcification [22]. All of the images extracted from in vivo pullbacks of the coronary artery. The Intravascular ultrasound imaging systems used for the acquisition is Si5 equipped with a $20 \mathrm{MHz}$ eagle eye monorail catheter. The image we analyzed is a cartesian coordinate image in a portable network graphic (PNG). The total images from dataset B would divide into two classes; calcification present and calcification absent. All the original size of the image were 384 by 384 with 1-channel. In this study, two types of images used to analyze the presence and absence of the calcification was the cartesian coordinate images and polar reconstructed coordinate images. The second image, the polar reconstructed coordinate image, was obtained by transforming the cartesian coordinate image using the daugman rubber sheet normalization model. After transformation, the polar reconstructed images are resized to 384 by 384 , similar to the cartesian coordinate image. Figure 2 showed the image transformation on the intravascular ultrasound image using the daugman rubber sheet normalization model [18].

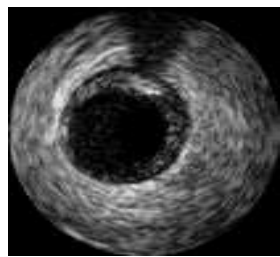

(a)

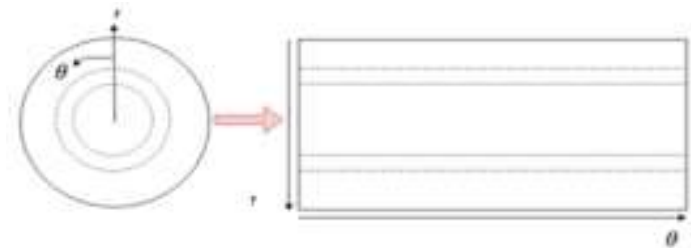

(b)

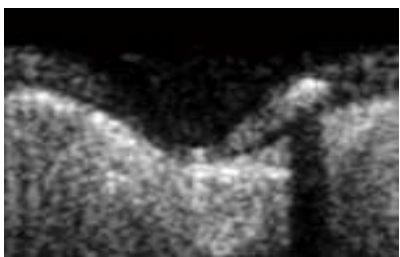

(c)

Figure 2. (a) Cartesian coordinate image; (b) The transformed model using rubber sheet normalization model; (c) Polar reconstructed coordinate image [18]

\subsection{Proposed architecture}

Figure 3(a) shows the proposed 27 layers of convolutional neural networks (CNNs) using direct acyclic graph (DAGNet-27). The design was suitable to be used for an IVUS image of size 384 by 384 with 
1- channel. The input image has been built together with max pool, batch normalization and ReLu whilst the convolutional also was built together with max pool, batch normalization and ReLU. The DAGNet-27 has 27 layers with net properties, [27x1 nnet.cnn.layer.Layer]; Connections: [27x2 table]. In Figure 3(a), there are six convolutions layers by layers used, 3rd convolutions layers by layers show that the algorithm used transfer learning. Figure 3(b) shows the convolutional layers built together with the max pool, batch normalization and ReLu layer. In the CNNs architecture proposed, we did not use dropout because the gap between the validations, training and smoothing is minimal. We choose the max pool without overlapping to get a better edge for calcification detection. This proposed CNNs architecture was designed based on the size of the original IVUS images. The CNNs architecture proposed used 1-channel images as compared to standard CNN algorithms that used 3- channel images.

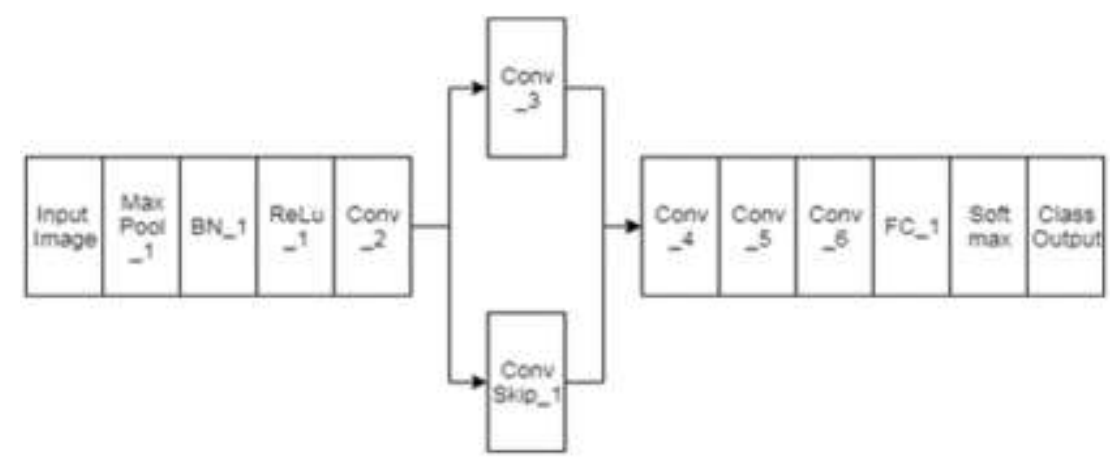

(a)

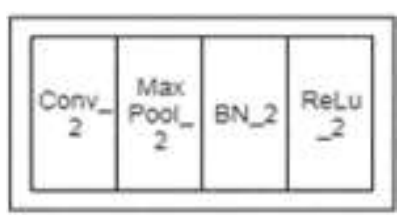

(b)

Figure 3. (a) Top - The DAGNet - 27 architecture with 6 - convolution layers and 1 - fully connected layer

(b) Bottom- the convolutional layers with maxpool layer, batch normalization layer and ReLu layer

Figure 4 shows the proposed framework block diagram for calcification detection using an original image of size 384 by 384 with 1-channel. First, all the image were resized to 384 by 384 with 1-channel, then all the image were separated into two classes, as CALC (Calcification presence) and as NoCALC (calcification absence). The next step was to feed the image to the DAGNet-27 architecture for further the deep feature learning process. The output layer is the fully connected layers together with the softmax layer and class output layer. Next, classification with cross-validation will perform using split. The value of the splitting used; 0.4999, 0.6666, 0.8 and 0.9. For the partitioning, the $\mathrm{k}$-fold formula are $\mathrm{TR}=(\mathrm{k}-1) / \mathrm{k}$ is the training and, $\mathrm{TE}=1 / \mathrm{k}$ is the testing [21]. The value of $\mathrm{k}$ is $2,3,5$ and 10 [21]. The $\mathrm{k}$-fold cross-validation is a procedure used to estimate the skill of the model on new data. The fold cross-validation is applied for this research to evaluate the predictive model by partitioning the original samples during testing and training. The reason is, the method provides adequate data for training and data for validation. The proposed model was pre-trained to save time and to get better performance. The training classifier for dataset B was assigned using labelling; 'CALC' and 'NoCALC' as define earlier. In the last step, the results obtained will compare with the ground truth [22].

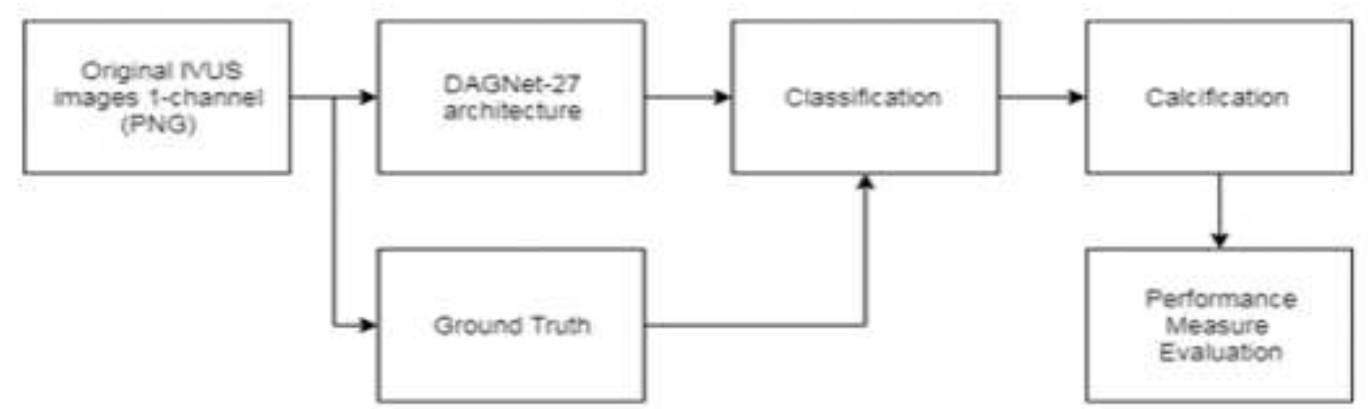

Figure 4. The framework of the proposed convolutional neural networks with 1-channel 


\subsection{Learnable parameters}

The learnable parameters were the sum of offset, scale, weight and biases. The non-learnable parameters are the activation of the height product, the filter width and the input channels number. The total learnable parameters of the $\mathrm{CNN}$ architecture are the total sums of the convolution layers and fully connected that have weight and biases. The non-learnable parameters are the product of image input size for the filter size and the numbers of channels. The total number of the convolutional layer parameters is $\left[\left(\mathrm{h}^{*} \mathrm{w}^{*} \mathrm{c}+\right.\right.$ 1)*Filters Numbers)], where ' 1 ' is the bias. The convolutional layer output size, is for the output height and width that is [(The size of the Input $-(($ The size of the Filter -1$) *$ Dilation Factor +1$)+2 *$ Padding $) /$ Stride + 1]. The total neuron number for the output size in a convolutional layer is the Map Size of product*Filters Numbers. The total parameters for the fully connected layer are the multiplication of the input by adding weight matrix, $\mathrm{W}$ and bias vector [23]. The non-overlapping max pool for 1-channel of the pool size and stride is the same. The input for the pooling layer is n-by-n. The h-by-h is the pooling layer down-samples the regions by $\mathrm{h}$. Therefore, the maximum output or average pooling region size is the layer for one channel of a convolutional layer that is $n / h-b y-n / h$ [24]. The total parameters obtained was 55668 .

\section{RESULTS AND DISCUSSION}

The results were shown in Table 1 and Table 2 for both types of images. The 1st column of the table is the $\mathrm{k}$-fold, taken value $\mathrm{k}$ as $2,3,5$ and 10 . Other data recorded are the outcomes from the confusion matrix, namely the true positive (TP), false positive (FP), true negative (TN), and false negative (FN). TP is the total calcification presence correctly detected, FP is the total calcification absence incorrectly detected as calcification presence, TN is the total calcification absence correctly detected and FP is the total calcification absence incorrectly detected as calcification presence? The derived measurements from the confusion matrix are also recorded. They are accuracy (Acc), sensitivity (Sn), specificity (Sp), positive predictive value (PPV) and negative predictive value (NPV). Accuracy is the ratio number of correct predictions to the total number of testing images. Sensitivity is the true positive rate and is defined as TP/(FN+TP). Specificity is a true negative rate and is defined as $\mathrm{TN} /(\mathrm{FP}+\mathrm{TN})$. PPV is the probability that the disease is presence given a positive test result and is defined as TP/TP+FP. Similarly, the NPV is the probability that the disease is absent given a negative test result, and is defined as TN/TN+FN [25].

Table 1 and Table 2 shows the cartesian coordinate images and polar reconstructed coordinate images performance respectively. For the cartesian coordinate images, the range value for 2 -fold is from $88.30 \%$ to $98.70 \%$, whilst the range value for 3 -fold is $93.80 \%$ to $98.90 \%$ and 5 -fold the range value is $94.30 \%$ to $98.80 \%$. The 10 -fold has shown an excellent performance value, ranging from $92.50 \%$ to $100.0 \%$. For Polar Reconstructed coordinate image, the range value for 2-fold is from $90.60 \%$ to $97.90 \%$, whilst the range value for 3 -fold is $93.20 \%$ to $99.50 \%$ and 5 -fold the range value is $97.20 \%$ to $99.70 \%$. The 10 -fold has shown a performance value ranging from $92.50 \%$ to $100.0 \%$. Table 2 shows the improvement results i.e. the performance measures such as the accuracy, sensitivity, specificity and, negative prediction value are better when the k-fold is larger when more images are used for training and fewer images are used for testing.

Table 1. The confusion matrix and performance measure evaluation of cartesian coordinate image

\begin{tabular}{cccccccccc}
\hline \multirow{2}{*}{ k-fold } & \multicolumn{3}{c}{ Confusion matrix (Testing) } & \multicolumn{4}{c}{ Performance Measure Evaluation (\%) } \\
\cline { 2 - 10 } & TP & TN & FP & FN & Acc & Sn & Sp & PPV & NPV \\
\hline 2 & 234 & 812 & 31 & 11 & 96.14 & 95.50 & 96.30 & 88.30 & 98.70 \\
3 & 166 & 542 & 11 & 6 & 97.66 & 96.50 & 98.00 & 93.80 & 98.90 \\
5 & 100 & 325 & 6 & 4 & 97.70 & 98.20 & 98.20 & 94.30 & 98.80 \\
10 & 49 & 164 & 4 & 0 & 98.16 & 100.00 & 97.60 & 92.50 & 100.00 \\
\hline
\end{tabular}

Table 2. The confusion matrix and performance measure evaluation of polar reconstructed coordinate image

\begin{tabular}{cccccccccc}
\hline \multirow{2}{*}{ k-fold } & \multicolumn{3}{c}{ Confusion matrix (Testing) } & \multicolumn{4}{c}{ Performance Measure Evaluation (\%) } \\
\cline { 2 - 10 } & TP & TN & FP & FN & Acc & Sn & Sp & PPV & NPV \\
\hline 2 & 240 & 806 & 25 & 17 & 96.14 & 93.40 & 97.00 & 90.60 & 97.90 \\
3 & 165 & 545 & 12 & 3 & 97.93 & 98.20 & 97.80 & 93.20 & 99.50 \\
5 & 103 & 328 & 3 & 1 & 99.08 & 99.00 & 99.10 & 97.20 & 99.70 \\
10 & 51 & 164 & 2 & 0 & 99.08 & 100.00 & 89.80 & 89.20 & 100.00 \\
\hline
\end{tabular}

Figure 5 and Figure 6 shows the training progress for 10-fold for cartesian coordinate image and polar reconstructed coordinate image respectively. The validation accuracy shows $100 \%$ with 10-epoch and 225 iterations with 15 iterations per epoch. The time taken was about 23 minutes 33 seconds for cartesian coordinate images and 23 minutes 41 seconds for polar reconstructed coordinate image. By using the pre-

Calcification detection for intravascular ultrasound image using direct acyclic graph... (Hannah Sofian) 
trained-model, Figure 4 shows the training progress for cartesian coordinate images and polar reconstructed coordinate images at the higher start of the performance whilst higher slope and higher asymptote for the performance versus training [26]. The final result is Table 3 and it shows the comparison result between the proposed method and other works in the literature.

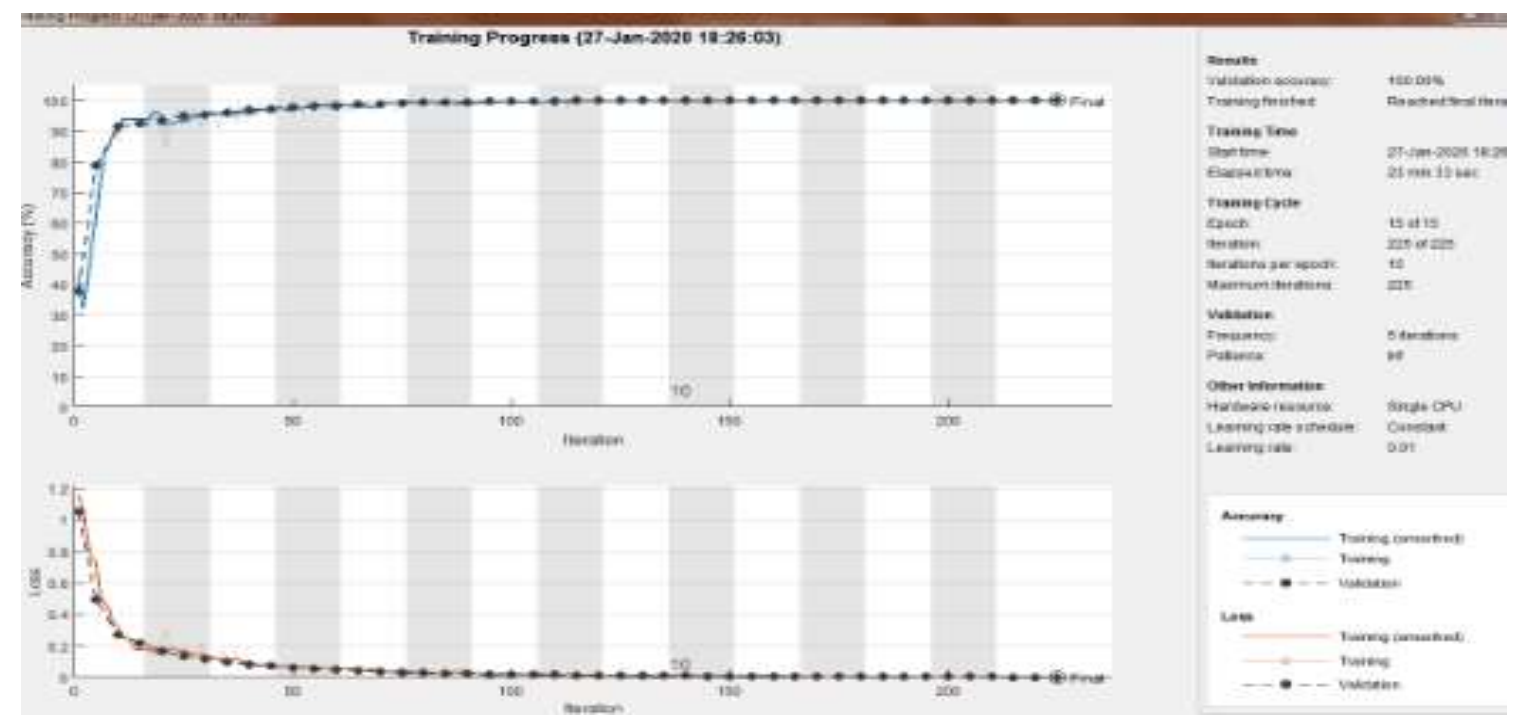

Figure 5. The proposed architecture training progress using transfer learning for 10-fold for cartesian coordinate image

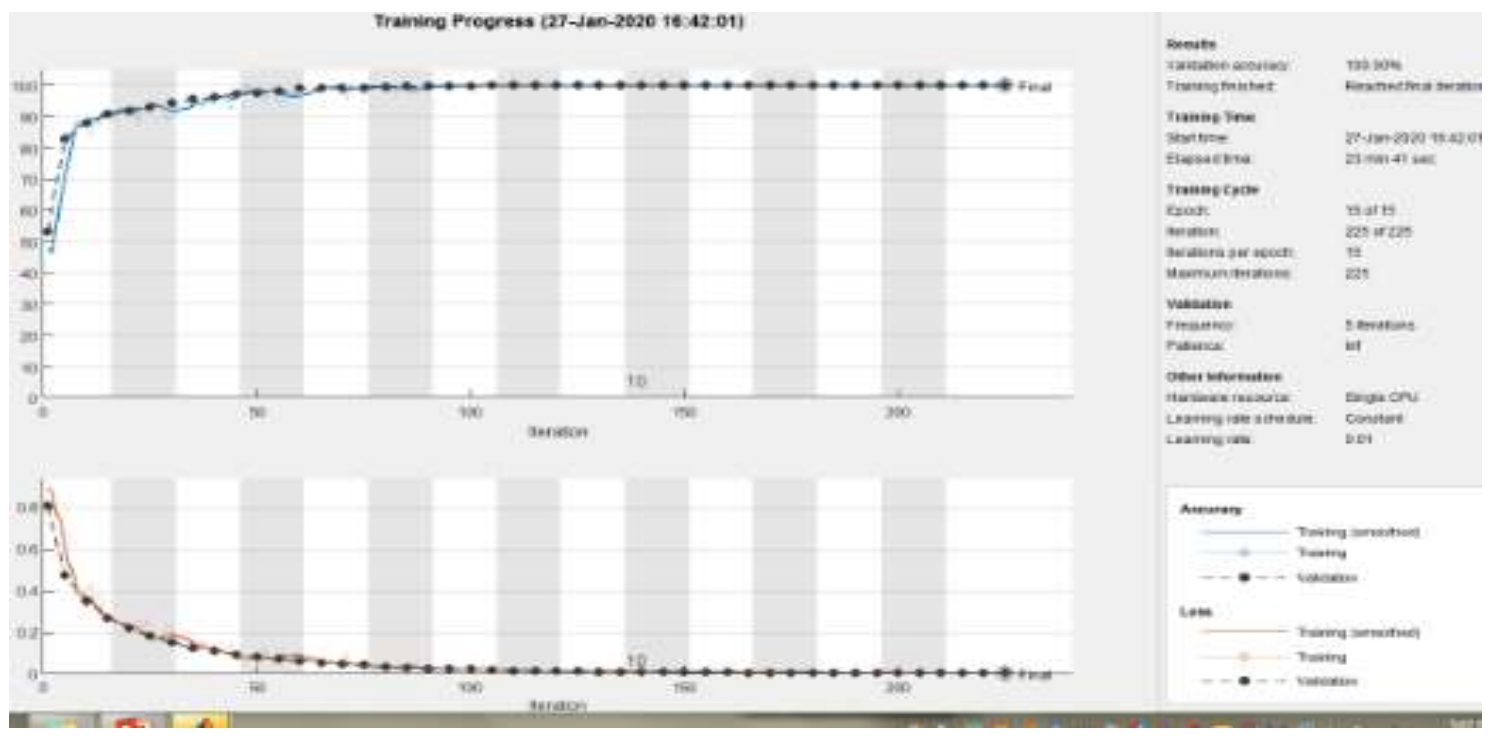

Figure 6. The proposed architecture training progress using transfer learning for 10-fold for polar reconstructed coordinate image

Table 3. The performance measures evaluation using deep learning

\begin{tabular}{|c|c|c|c|c|c|c|}
\hline Author & Diseases & $\operatorname{Acc}(\%)$ & $\mathrm{Sp}(\%)$ & $\operatorname{Sn}(\%)$ & $\mathrm{PPV}(\%)$ & NPV(\%) \\
\hline Proposed Architecture & $\begin{array}{l}\text { Coronary Artery Disease (IVUS) - Calcification } \\
\text { with the plaque }\end{array}$ & 99.08 & 100.00 & 89.80 & 89.20 & 100.00 \\
\hline Awais et al. 2018 & Diabetic Macular Edema (OCT) & 87.50 & 93.50 & 81.00 & - & - \\
\hline Gao et al. 2014 & Coronary Artery Disease (IVUS)-Calcified & - & 94.68 & 95.82 & - & - \\
\hline Taki et al. 2008 & Coronary Artery Disease (IVUS) - Calcification & - & 92.76 & 98.50 & - & - \\
\hline
\end{tabular}




\section{CONCLUSION}

The proposed convolutional neural network architecture shows that the results obtained from the polar reconstructed coordinates images are better as compared to the cartesian coordinate image. In this study, using a direct acyclic graph and transfer learning has shown that deep learning had better results to differentiate images with calcification presence and calcification absence. When compared with two types of images, both images had an excellent performance measure evaluation result in accuracy, $99.08 \%$ and $98.16 \%$, sensitivity, $89.80 \%$ and $97.60 \%$ whilst negative predictive value, $89.20 \%$ and $92.50 \%$ and the perfect performance measure evaluation result in sensitivity and negative prediction value were $100 \%$.

\section{ACKNOWLEDGEMENTS}

We would like to thanks to Professor Dr Simone Balocco, Computer Vision Center, Dept. Matemàtica Aplicada i Anàlisi, Bellaterra-Universitat de Barcelona, Barcelona, for his cooperation in this study. The research funded by the Ministry of Higher Education Malaysia, Universiti Teknologi Malaysia Research Grant (Q.K. 130000.2540.14H22) and Universiti Kuala Lumpur.

\section{REFERENCES}

[1] T. Ziegler, F. Abdel Rahman, V. Jurisch, and C. Kupatt, "Atherosclerosis and the capillary network; pathophysiology and potential therapeutic strategies," Cells, vol. 9, no. 1, p. 50, 2020, doi: 10.3390/cells9010050.

[2] W. H. Organization, "World health statistics 2018: monitoring health for the SDGs, sustainable development goals," World Health Organization, 2018.

[3] W. Liu, Y. Zhang, C.-M. Yu, Q.-W. Ji, M. Cai, Y.-X. Zhao, and Y.-J. Zhou, "Current understanding of coronary artery calcification," Journal of geriatric cardiology (JGC), vol. 12, no. 6, p. 668, 2015, doi: 10.11909/j.issn.16715411.2015.06.012.

[4] M. S. Lo-Kioeng-Shioe, et al., "Coronary calcium characteristics as predictors of major adverse cardiac events in symptomatic patients: insights from the core 320 multinational study," Journal of the American Heart Association, vol. 8, no. 6, p. e007201, 2019, doi: 10.1161/JAHA.117.007201.

[5] S. K. Sharma, Y. Vengrenyuk, and A. S. Kini, "Ivus, oct, and coronary artery calcification: Is there a bone of contention?", 2017, doi: 10.1016/j.jcmg.2017.06.008.

[6] A. Taki, Z. Najafi, A. Roodaki, S. K. Setarehdan, R. A. Zoroofi, A. Konig, and N. Navab, "Automatic segmentation of calcified plaques and vessel borders in ivus images," International Journal of Computer Assisted Radiology and Surgery, vol. 3, no. 3, pp. 347-354, 2008, doi: 10.1007/s11548-008-0235-4.

[7] N. Dey, A. B. Roy, P. Das, A. Das, and S. S. Chaudhuri, "Detection and measurement of arc of lumen calcification from intravascular ultrasound using harris corner detection," in 2012 National Conference On Computing and Communication Systems. IEEE, 2012, pp. 1-6, doi: 10.1109/NCCCS.2012.6413021.

[8] X. Zhang, C. R. McKay, and M. Sonka, "Tissue characterization in intravascular ultrasound images," IEEE Transactions on Medical Imaging, vol. 17, no. 6, pp. 889-899, 1998, doi: 10.1109/42.746622.

[9] H. Sofian, A. Ng, J. Than, S. Mohamad, and N. M. Noor, "Calcification boundary detection in coronary artery using intravascular ultrasound images," in TENCON 2017-2017 IEEE Region 10 Conference. IEEE, 2017, pp. 2835-2839, doi: 10.1109/TENCON.2017.8228344.

[10] E. Dos Santos Filho, Y. Saijo, A. Tanaka, T. Yambe, S. Li, and M. Yoshizawa, "Automated calcification detection and quantification in intravascular ultrasound images by adaptive thresholding, " in World Congress on Medical Physics and Biomedical Engineering 2006. Springer, 2007, pp. 1421-1425, doi: 10.1007/978-3-540-36841-0_348.

[11] Z. Gao, W. Guo, X. Liu, W. Huang, H. Zhang, N. Tan, W. K. Hau, Y.-T. Zhang, and H. Liu, "Automated detection framework of the calcified plaque with acoustic shadowing in ivus images," PloS one, vol. 9, no. 11, p. e109997, 2014, doi: 10.1371/journal.pone.0109997.

[12] A. S. Lundervold and A. Lundervold, "An overview of deep learning in medical imaging focusing on mri," Zeitschrift fur Medizinische Physik, vol. 29, no. 2, pp. 102-127, 2019, doi: 10.1016/j.zemedi.2018.11.002.

[13] D. R. Dtv and K. Ramana, "A novel approach for efficient training of deep neural networks, ” Indonesian Journal of Electrical Engineering and Computer Science, vol. 11, pp. 954-961, 2018.

[14] F. Zaman, J. Johari, and A. Yassin, "Learning face similarities for face verification using hybrid convolutional neural networks," Indonesian Journal of Electrical Engineering and Computer Science, vol. 16, no. 3, p. 1333, 2019, doi: 10.11591/ijeecs.v16.i3.pp1333-1342.

[15] M. Awais, H. M"uller, and F. Meriaudeau, "Classification of sd-oct images using deep learning approach," IEEE International Conference on Signal and Image Processing Applications (ICSIPA). IEEE, 2017, doi: 10.1109/ICSIPA.2017.8120661.

[16] R. Kamble, G. Chan, O. Perdomo Charry, M. Kokare, H. M"uller, and F. Meriaudeau, "Automated diabetic macular edema (dme) analysis using fine tuning with inception-resnet-v2 on oct images," IEEE-EMBS Conference on Biomedical Engineering and Sciences (IECBES). IEEE, 2018, doi: 10.1109/IECBES.2018.8626616.

[17] A. Abdolmanafi, L. Duong, N. Dahdah, I. Adib, and F. Cheriet, "Characterization of coronary artery pathological formations from oct imaging using deep learning," Biomedical Optics Express, vol. 9, no. 10, p. 4936, 2018, doi: 10.1364/BOE.9.004936. 
[18] P. Xi, C. Shu, and R. Goubran, “Abnormality detection in mammography using deep convolutional neural networks," IEEE International Symposium on Medical Measurements and Applications (MeMeA). IEEE, 2018.

[19] T. A. M. Tengku Zainul Akmal, J. Than, H. Abdullah, and N. Noor, "Chest x-ray image classification on common thorax diseases using glcm and alexnet deep features," International Journal of Integrated Engineering, vol. 11, no. 4, 2019, doi: 10.30880/ijie.2019.11.04.003.

[20] D. Ragab, M. Sharkas, S. Marshall, and J. Ren, "Breast cancer detection using deep convolutional neural networks and support vector machines," PeerJ, vol. 7, p. e6201, 2019, doi: 10.7717/peerj.6201.

[21] H. Sofian, J. Ming, S. Mohammad, and N. Noor, "Calcification detection using convolutional neural network architectures in intravascular ultrasound images," Indonesian Journal of Electrical Engineering and Computer Science, vol. 17, pp. 1313-1321, 2020, doi: 10.11591/ijeecs.v17.i3.pp1313-1321.

[22] S. Balocco, C. Gatta, et al., "Standardized evaluation methodology and reference database for evaluating ivus image segmentation," Computerized Medical Imaging and Graphics, vol. 38, no. 2, pp. 70-90, 2014, doi: 10.1016/j.compmedimag.2013.07.001.

[23] C. Szegedy, V. Vanhoucke, S. Ioffe, J. Shlens, and Z. Wojna, "Rethinking the inception architecture for computer vision," in Proceedings of the IEEE conference on computer vision and pattern recognition, 2016, pp. 2818-2826, doi: 10.1109/CVPR.2016.308.

[24] J. Nagi, et al,. "Max-pooling convolutional neural networks for vision-based hand gesture recognition," 2011, pp. 342-347, doi: 10.1109/ICSIPA.2011.6144164.

[25] H. Sofian, J. C. M. Than, S. Mohammad, and N. M. Noor, "Calcification detection of coronary artery disease in intravascular ultrasound image: Deep feature learning approach," International Journal of Integrated Engineering, vol. 10, no. 7, 2018, doi: 10.30880/ijie.2018.10.07.005.

[26] S. J. Pan, “Transfer learning." p. 537-570, 2014.

\section{BIOGRAPHIES OF AUTHORS}

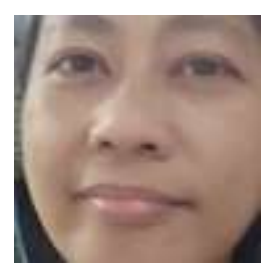

Hannah Sofian is currently in her final semester in $\mathrm{PhD}$ (Electrical Engineering) from Razak Faculty of Technology and Informatics, Universiti Teknologi Malaysia. She received her B. Eng. in Electrical and Electronics Engineering from West of Scotland University in Paisley, Scotland, Master of Science in Mechatronics (Signal and Systems) from International Islamic University Malaysia and. her current work concentrates on medical image analysis for coronary artery diseases in deep learning.

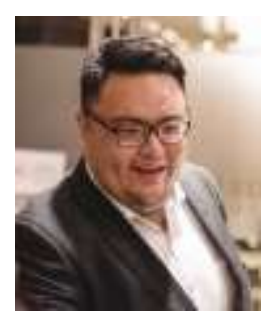

Dr Joel Chia Ming Than is currently attached as Senior Lecturer in School of Information and Communication Technologies, Faculty of Engineering, Computing and Science, Swinburne University of Technology Sarawak Campus, Malaysia received his $\mathrm{PhD}$ and Master of Philosophy from Universiti Teknologi Malaysia. He received B. Eng in Biomedical Engineering from Universiti Tunku Abdul Rahman. He is passionate is in medical imaging, machine learning and deep learning.

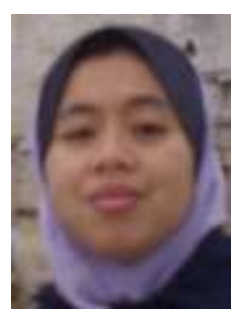

Dr Suraya Mohammad is a Senior Lecturer in Universiti Kuala Lumpur, British Malaysian Institute. She graduated with a Bachelor degree in Engineering from the University of Tasmania, Australia, completed an MSc degree in Mechatronic at the IIUM and was awarded a PhD degree in Computer Science from The University of Manchester, UK. Her research interest is in data communication, networking and, signal and image processing with application in biomedical and computer vision.

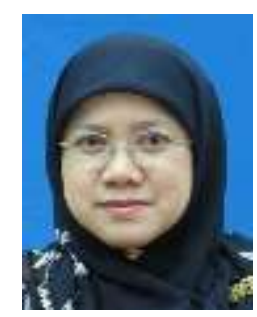

Professor Dr Norliza Mohd Noor is currently attached with Razak Faculty of Technology and Informatics, Universiti Teknologi Malaysia (UTM), Kuala Lumpur Campus. She received her B.Sc. In Electrical Engineering from Texas Tech University in Lubbock, Texas and her Master of Electrical Engineering (by research) and $\mathrm{PhD}$ (Electrical Engineering) from UTM. Her research area is in image processing and image analysis. Her current work concentrates on medical image analysis for lung diseases. Currently, she is the Head of the Electrophysiology Research Group, UTM Razak Faculty of Technology and Informatics. 